Preisverleihung

Implantatforschungspreis 2012 verliehen

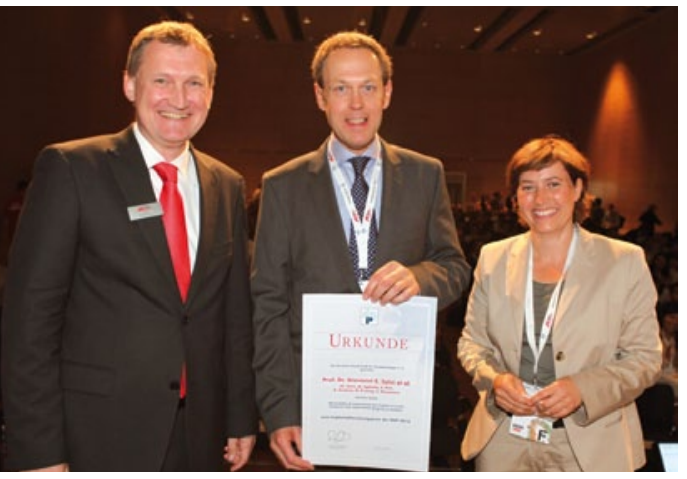

Der Implantatforschungspreis 2012 der Deutschen Gesellschaft für Parodontologie (DGP) ging an Prof. Giovanni E. Salvi, Bern. Er wurde für die wissenschaftliche Arbeit mit dem Thema „Reversibility of experimental peri-implant mucositis compared with experimental gingivitis in humans" ausgezeichnet. Die Publikation verfasste der Schweizer mit 5 weiteren Co-Autoren. „Wir freuen uns jedes Jahr über das hohe wissenschaftliche Know-how der eingereichten Arbeiten“,
V.I.n.r.: Dr. Ralf Rauch, Geschäftsführer Nobel Biocare Deutschland $\mathrm{GmbH}$, der Preisträger Prof. Giovanni E. Salvi und PD Dr. Bettina Dannewitz, Mitglied des Vorstands der DGP.

so Dr. Ralf Rauch, Geschäftsführer der Nobel Biocare Deutschland $\mathrm{GmbH}$, und weiter: „Wir benötigen die Erkenntnisse wissenschaftlicher Studien, wie sie der Preisträger vorgestellt hat, um Standards für die Periimplantatitis-Therapie zu entwickeln.“ Der Preis wurde im Rahmen des Corporate Forums der Nobel Biocare auf der Europerio 7 im Juni dieses Jahres in Wien verliehen. Der Implantatforschungspreis zeichnet richtungsweisende wissenschaftliche Arbeiten im Bereich der Implantattherapie aus, die in einem internationalen peer-reviewed Journal veröffentlicht worden sind. Das Unternehmen fördert den Preis seit mehreren Jahren. Er ist mit 5000,- € dotiert.

Nach einer Pressemitteilung der Nobel Biocare Deutschland GmbH, Köln Internet: www.nobelbiocare.com

\title{
Wechsel im Management
}

Materialise Dental, mit dem SimPlant ${ }^{\circledR}$ System der führende Anbieter 3-dimensionaler digitaler Implantologie, hat einen Wechsel im Management bekannt gegeben: Dr. Raquel Neumann hat mit Wirkung zum 1. August 2012 die Aufgabe der Country Managerin übernommen und ist damit für Deutschland, Österreich und die deutschsprachige Schweiz verantwortlich. In ihrer neuen Rolle setzt die promovierte Zahnmedizinerin auf hochwertigen Service und eine umfassende Kundenbetreuung. Als neue Country Managerin hat es sich Neumann zur Aufgabe gemacht, die Position des Unternehmens im Bereich computernavigierte Implantologie weiter auszubauen. Ein besonderes Augenmerk legt Neumann dabei auf das Serviceangebot:
Dazu gehört nicht nur der Ausbau starker Partnernetzwerke, etwa mit Herstellern im Bereich der Implantologie und Radiologie, sondern auch die Sicherstellung einer umfassenden Kundenberatung. Neumann kann bereits auf langjährige Erfahrungen zurückblicken. Nach dem Studium der Zahnmedizin in Brasilien zog es die gebürtige Argentinierin nach Deutschland. Hier promovierte Neumann zunächst an der LMU, bevor sie sich auf Unternehmensseite neuen Herausforderungen stellte: Von der Heraeus Kulzer $\mathrm{GmbH}$, bei der sie im Produktmanagement tätig war, wechselte Neumann 2009 zu DENTSPLY Friadent und zeichnete als Global Brand Managerin Digital Dentistry für den Bereich Guided Surgery und CAD/CAM verantwortlich. Parallel dazu erlangte sie 2011 an der renommierten Mannheimer Business School ihren Executive Master of Business Administration.

Nach einer Pressemitteilung der

Materialise Dental GmbH, Gilching

Internet: www.simplant.de

\section{Gut gerüstet für die Praxis}

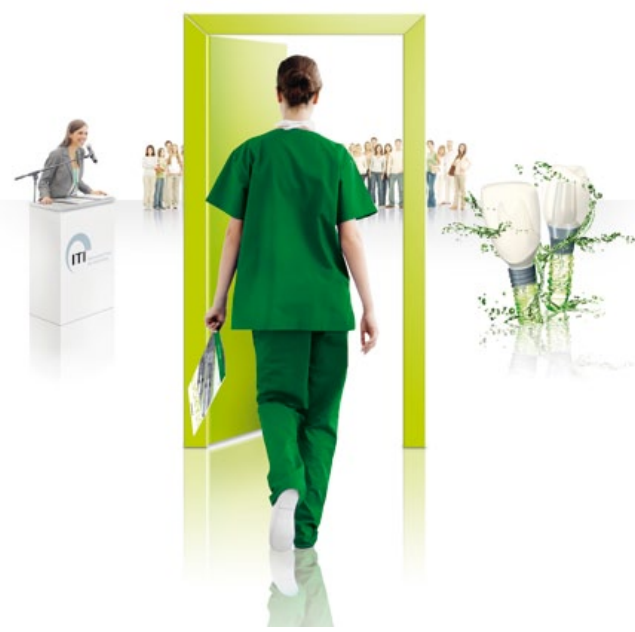

Bereits während ihres Studiums gehen angehende Zahnärzte die ersten wichtigen Schritte in Richtung Karriereleiter. Umso wichtiger ist es, von Anfang an den Durchblick zu behalten und Grundsteine richtig zu legen. Mit dem Young Professional Program unterstützt die Straumann GmbH junge Zahnmediziner bei dieser besonderen Herausforderung und begleitet sie auf ihrem Weg in die berufliche Zukunft. Und zwar vom 8. Semester an über ihre Assistenzzeit bis hin zur Gründung ihrer eigenen Zahnarztpraxis oder einer Praxisübernahme.

Im Rahmen des Programms erhalten Young Professionals Leistungen, die maßgeschneidert zu ihrem Einstieg ins Berufsleben passen. Dazu zählen Sonderkonditionen für Fortbildungsveranstaltungen und ausgewählte Fachliteratur, Beratungsunterlagen zur Praxisgründung sowie Einsteiger-Kits in die Chirurgie und Implantatprothetik.

Die im Programm enthaltenen Leistungen gehen auf alle Anforderungen und Bereiche einer modernen Zahnarztpraxis ein: mit Fortbildungen und Kursen, mit Events und regelmäßigen aktuellen Informationen zu Produkt- und Servicethemen sowie durch eine individuelle Betreuung vor Ort.

Interessierte können sich direkt online zum Straumann Young Professional Program anmelden und erhalten unverbindlich zur Begrüßung ihr persönliches Starterpackage: http://www.straumann.de/ de/ypp.html.

Nach einer Pressemitteilung der

Straumann GmbH, Freiburg 


\section{Viel Lob für die Deutsche Gesellschaft für Orale Implantologie}

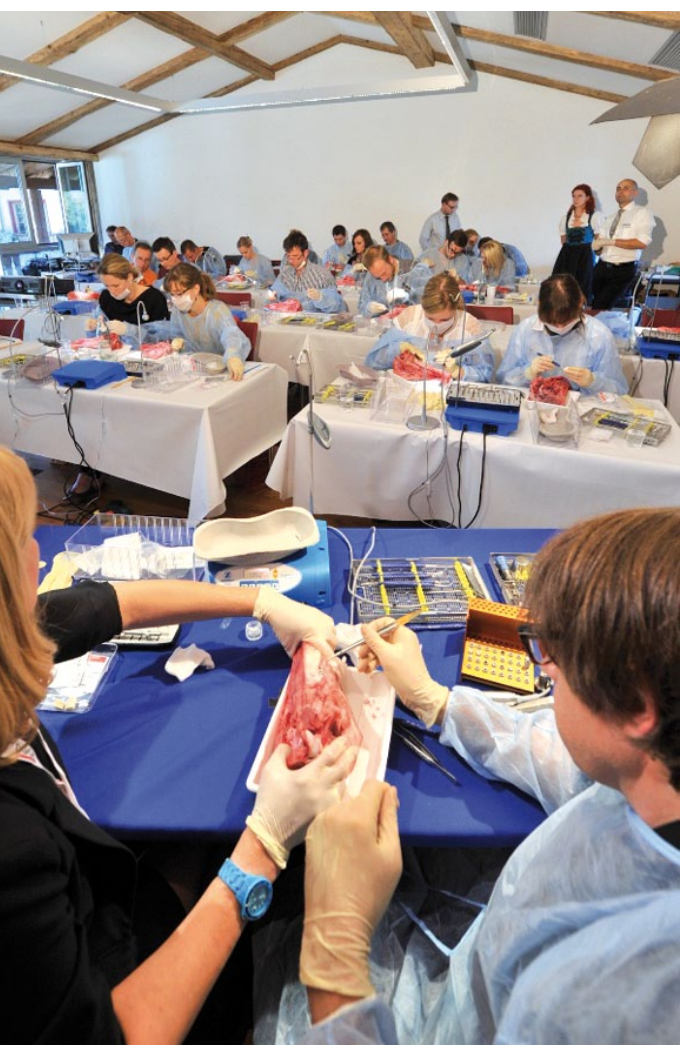

„Es macht Spaß in kleineren Gruppen zu arbeiten, weil ein intensiverer Austausch mit den Referenten möglich ist.“ „Meine Arbeit kann ich nur durch das praxisorientierte Training verbessern. Deshalb finde ich dieses Workshop-Konzept gelungen."

So begeistert äußerten sich die meisten der rund 200 Teilnehmer über den 9. Jahreskongress der DGOI in Grassau/ Chiemgau, den die Fachgesellschaft vom 3.-6. Oktober zum 1. Mal als WorkshopKongress veranstaltete. Dem Motto „PraWissimo - 100 Prozent Praxisrelevanz auf wissenschaftlicher Basis“ entsprach das Programm voll und ganz.

Von Mittwoch bis Freitag gab es mehr als 20 verschiedene Workshops und Seminare, sodass sich die Teilnehmer - ihren individuellen Interessen entsprechend - ein Trainingsprogramm mit vielen Hands-On-Übungen zusammenstellen konnten. Das Mainpodium am Samstag stand ganz im Zeichen des wissenschaftlichen Austauschs, mit Themen rund um Geweberegeneration und -aufbau sowie
Gewebestabilität und -erhalt am Implantat.

„Wir haben das neue Konzept als eine Art Probelauf gestartet und freuen uns sehr, dass es bei den Teilnehmern sehr gut angekommen ist“, so Dr. Georg Bayer, Präsident der DGOI und weiter: „Deshalb werden wir dieses Konzept in 2 Jahren wiederholen.“ Zusätzlich zu den zahlreichen Workshops der Industriepartner fanden Kompetenzworkshops zu 5 verschiedenen Themen statt, die DGOI-Fortbildungsreferent Prof. Georg-H. Nentwig eigens für diesen Kongress entwickelt hat. $\mathrm{Zu}$ spüren war während der gesamten Kongresstage die familiäre und freundschaftliche Atmosphäre unter den Teilnehmern und Referenten. Genügend Zeit für den kollegialen Austausch hatten die Zahnärzte und Zahntechniker während des Get-togethers in der Dentalausstellung am Donnerstagabend und dem zünftigen bayerischen Abend am Freitag.

Nach einer Pressemitteilung der

DGOI Büro, Kraichtal

Internet: www.dgoi.info

\section{Implantatpatienten gut informieren}

Über 50\% aller dentalen Implantate sind langfristig von Entzündungen bedroht, die sogar zum Verlust des Implantats führen können. Eine sorgfältige häusliche Mundhygiene der Implantatpatienten und ein strukturiertes Recall-System in der Praxis können diesen Entzündungen vorbeugen. Für die entsprechende Patientenaufklärung ist die begehrte Broschüre "Implantate brauchen Pflege“ ab sofort wieder beim Aktionsbündnis gegen Periimplantitis erhältlich.

Die Erstauflage der Broschüre „Implantate brauchen Pflege“, die Zahnarztpraxen bei der Patientenaufklärung über die richtige Pflege von Implantaten unterstützt, war bereits wenige Wochen nach Erscheinen vergriffen. Die nun erhältliche, weiter verbesserte 2 . Auflage erklärt auf 8 übersichtlichen Seiten im DIN-A5Format leicht verständlich Mechanismen in der Mundhöhle und gibt praktische Tipps zur Implantatreinigung, ohne dabei Ängste zu schüren. Die patientengerechte
Gestaltung macht die Broschüre zu einem hochwertigen Aufklärungsmedium in jeder Zahnarztpraxis.

Erstellt wurde die Broschüre „Implantate brauchen Pflege“ von der Arbeitsgruppe gesundes Implantat in Zusammenarbeit mit dem wissenschaftlichen Beirat des Aktionsbündnisses gegen Periimplantitis. Interessierte Zahnmediziner können die Patientenbroschüre gegen eine Schutzgebühr unter www.gegen-periimplantitis.de bestellen oder ein GratisPDF anfordern. „Die Nachfrage nach der 2. Auflage von Implantate brauchen Pflege ist bereits jetzt sehr hoch. Uns haben in den vergangenen Wochen viele Vorbestellungen von interessierten Praxen erreicht. Das bestätigt uns in unserer Arbeit gegen periimplantäre Entzündungen und zeigt deutlich, wie dringend derartige Aufklärungsmedien benötigt werden“, erklärt Jan-Philipp Schmidt, Initiator des Aktionsbündnisses gegen Periimplantitis.

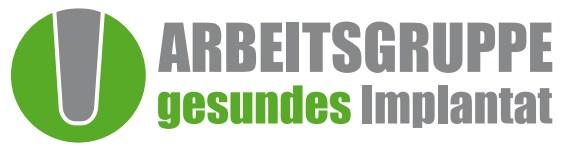

Über das Aktionsbündnis

Im Aktionsbündnis gegen Periimplantitis engagieren sich namhafte Unternehmen aus den Bereichen der Implantologie und Prophylaxe für die Prävention periimplantärer Entzündungen. Der wissenschaftliche Beirat und führende Fachverlage unterstützen das Bündnis bei seiner Arbeit. Die Arbeitsgruppe gesundes Implantat realisiert die Patientenkommunikation des Aktionsbündnisses und hat in dieser Funktion die Broschüre „Implantate brauchen Pflege“ erstellt. Mit der 1. Auflage der Broschüre konnte im Juni dieses Jahres erstmals über Unternehmensgrenzen hinweg ein fachlicher Konsens zur Pflege von Implantaten gefunden werden.

Nach einer Pressemitteilung des Aktionsbündnis gegen Periimplantitis, c/o DentaMedica GmbH, Leipzig 